Präv Gesundheitsf 2022 · 17:1-6 https://doi.org/10.1007/s11553-021-00840-1

Eingegangen: 8. November 2020

Angenommen: 15. Februar 2021

Online publiziert: 16. März 2021

(c) Der/die Autor(en) 2021

\author{
Thomas Niewöhner ${ }^{1,2}(\mathbb{D}) \cdot$ Leonie Hoerdler $^{1} \cdot$ Alina Roxel $^{1} \cdot$ Merle Meindl $^{1}$. \\ Ziwen Teuber ${ }^{1,2}$ \\ 'Universität Bielefeld, Bielefeld, Deutschland \\ ${ }^{2}$ Fakultät für Psychologie und Sportwissenschaften, Abteilung für Psychologie, Universität Bielefeld, \\ Bielefeld, Deutschland
}

\title{
Zielorientierung, Burnout und Engagement im Hochschulkontext: Integration von Zielorientierung in das Study Demands-Resources-Modell
}

schöpfung. Sie bewerten die Anforderungen als höher und Zeitspielräume als deutlich geringer [17]. Steigende persönliche Kosten in Form von Studienabbrüchen, psychischen Erkrankungen und damit verbundenen gesamtgesellschaftliche Konsequenzen wie ökonomische Fehlinvestitionen [3] sind die Folge. Von Interesse scheinen daher insbesondere Faktoren zu sein, welche den studienbezogenen Anforderungen entgegenwirken und die Bewältigung des Studienalltags erleichtern. Eine zentrale personale Ressource stellt dabei die studienbezogene Motivation dar. Dabei sind Zielorientierungen (als dispositionale Handlungsabsicht) von zentraler Bedeutung, da sie die Wahrnehmung von Leistungssituationen und kompetenzbasiertem Verhalten beeinflussen [6]. In der vorliegenden Studie werden hierzu Zielorientierungen als personale Ressource in das etablierte "Study Demands-Resources-Modell“ (SD-R-Modell) integriert, welches Erklärungsansätze dafür bietet, warum einige Studierende bei hohen Anforderungen mit Erschöpfung reagieren und andere engagiert bleiben [10, 13].

\section{Das SD-R-Modell}

Der Ursprung für das SD-R-Modell ist das für den Arbeitskontext entwickelte „Job Demands-Resources-Modell“ (JDR-Modell; z. B. [1, 2]). Das Modell erklärt die Entstehung von Burnout und Engagement aufgrund des direkten und indirekten Zusammenwirkens von arbeitsbzw. studienbezogenen Anforderungen und Ressourcen.

Burnout wird dabei durch die Dimensionen emotionale Erschöpfung, Zynismus und reduziertes Wirksamkeitserleben charakterisiert. Emotionale Erschöpfung ist das Initialsymptom von Burnout und führt bei anhaltenden Anforderungen zur Abwertung des Studiums sowie zu reduzierter Selbstwirksamkeit [24]. In dieser Studie wird daher emotionale Erschöpfung als das zentrale Symptom des Burnouts fokussiert. Engagement stellt den Gegenpol zu Burnout dar und beschreibt den Einsatz und die Energie, welche im Studium aufgewendet werden. Engagement ist durch einen positiven Zustand von Vitalität, Hingabe und Vereinnahmung [20] gekennzeichnet. Die Anforderungen des Studienumfelds beziehen sich auf alle physischen, psychischen, sozialen und organisationalen Aspekte, die eine Bewältigung durch das Individuum erfordern (z.B. Zeitdruck, ein hohes Maß an Konzentration oder die Bewältigung mehrerer Aufgaben zur gleichen Zeit). Die Ressourcen kennzeichnen Aspekte, die dabei helfen, die studienbezogenen Anforderungen $\mathrm{zu}$ bewältigen (z.B. Unterstützung durch Lehrende und Kommilitonen, oder die und psychischen Beschwerden und zei-
gen vermehrt Anzeichen emotionaler Er- 


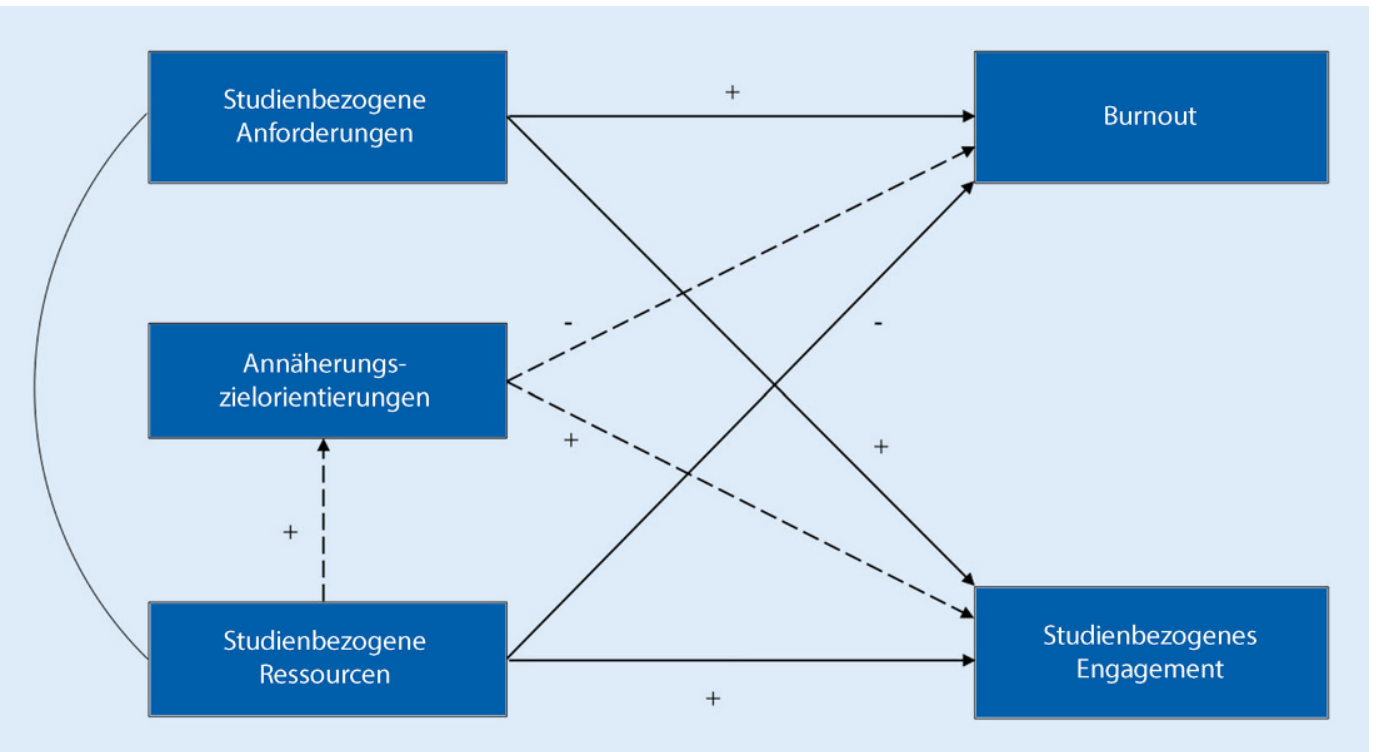

Abb. $1<$ SD-R-Modell (Study Demands-Resources-Modell) angelehnt an Lesener et al. [13] mit Integration der Annäherungszielorientierung

Möglichkeit sinnvolle Qualifikationen für die eigene Zukunft zu erlangen; [13]).

Bezogen auf diese Variablen bildet das Modell zwei parallele, direkte psychologische Prozesse ab: Zum einen führt ein gesundheitsbeeinträchtigender Prozess dazu, dass durch zu hohe, dauerhafte Anforderungen mentale und physische Ressourcen ausgeschöpft werden. Dies kann zu Burnout und langfristig zu gesundheitlichen Problemen (z. B. Depression, Herzkreislaufstörungen, Substanzabhängigkeiten) führen [14]. Zum anderen wird ein motivationaler Prozess angenommen, in dem die Studienressourcen durch ihr motivationales Potenzial zu Engagement und langfristig zu positiven psychischen und akademischen Outcomes führen (z. B. geringerem Risiko eines Studienabbruchs, höheres Wohlbefinden; [18]).

\section{Personale Ressourcen im SD-R- Modell}

Das ursprüngliche JD-R-Modell wurde um die Variable der personalen Ressourcen erweitert. Personale Ressourcen sind protektive Faktoren, welche einen adaptiven Umgang mit Anforderungen der Umwelt ermöglichen und zu einer positiven Entwicklung des Individuums beitragen [11]. Personale Ressourcen können adaptive charakterliche, kognitive sowie motivationale Eigenschaften von Perso- nen sein. Erste empirische Befunde hierzu lieferten Xanthopoulou et al. [25], indem sie Ansatzpunkte für eine vermittelnde Wirkung personaler Ressourcen im JD-R-Modell fanden. Es zeigte sich, dass die Eigenschaften Selbstwirksamkeit, organisationsbezogener Selbstwert und Optimismus den Zusammenhang zwischen Jobressourcen und Engagement sowie Burnout mediierten.

Im SD-R-Modell wurden studienbezogene Ressourcen, wie z.B. Unterstützung durch Lehrende und Kommilitonen, bereits berücksichtigt [13]. Personale Ressourcen hingegen wurden bisher noch nicht ausreichend untersucht und in das Modell integriert. Besonders individuelle Unterschiede bezüglich motivationaler Aspekte fanden in der deutschen Hochschulforschung im Zusammenhang von Burnout und Engagement bisher wenig Beachtung, obwohl bereits erste Ansatzpunkte für motivationale Wirkungen gefunden werden konnten [16,21]. In der vorliegenden Studie werden deshalb Zielorientierungen als personale Ressource im Kontext des SD-R-Modells untersucht.

\section{Zielorientierungen als personale Ressource}

Im Hochschulkontext spielen besonders leistungsbezogene Anforderungen eine große Rolle. Wie diese Anforderungen bewältigt werden, kann von der verfolgten Zielorientierung der Studierenden abhängen (z.B. [5]). Zielorientierungen (definiert als motivationale Absichten, ein bestimmtes Verhalten $\mathrm{zu}$ zeigen) scheinen als personale Ressource von zentraler Bedeutung zu sein, da sie die Wahrnehmung von Leistungssituationen und kompetenzbasiertem Verhalten beeinflussen. In diesem Zusammenhang wurde ein $2 \times 2$-Rahmenmodell entwickelt, das aus Annäherungslern-, Vermeidungslern-, Annäherungsleistungs- und Vermeidungsleistungszielorientierungen besteht [6]. Bei Lernzielen geht es darum, die eigene Kompetenz weiterzuentwickeln, während es bei Leistungszielen darum geht, im Vergleich zu anderen bessere Leistungen $\mathrm{zu}$ erbringen. Im Vordergrund steht entweder, sich wünschenswerten Möglichkeiten anzunähern oder negative Möglichkeiten zu vermeiden.

Die unterschiedlichen Zielorientierungen werden mit unterschiedlichen emotionalen und behavioralen Konsequenzen adaptiver und maladaptiver Art in Verbindung gebracht. Während Vermeidungsziele protektiv (eher maladaptiv) eingesetzt werden, um Selbstwertverluste $\mathrm{zu}$ verhindern, werden Annäherungsziele aktiv verwendet, um Ressourcen aufzubauen und so einen adaptiven Umgang mit der Umgebung zu ermöglichen. In der vorliegenden Studie werden daher die mit Resilienz assoziierten Annäherungszielorientierungen 
betrachtet. Belege fanden u. a. TuominenSoini et al. [22]. Sie konnten herausstellen, dass Annäherungstendenzen positiv mit subjektivem Wohlbefinden zusammenhingen, während Vermeidungstendenzen zu Anpassungsproblemen bei Schülern führten. Ähnliche Ergebnisse lieferten Studien von Shih [21] und Naidoo et al. [16]. Sie brachten Annäherungszielorientierungen in einen positiven Zusammenhang mit Engagement und einen negativen Zusammenhang mit Burnout [21]. Außerdem stellten sie einen positiven Zusammenhang von Vermeidungszielen und Burnout, sowie einen negativen Zusammenhang zwischen Annäherungszielen und Burnout fest. Die Autoren betonten die Rolle der Valenz (Annäherung oder Vermeidung) und vermuteten, dass Annäherungsorientierungen als ein Puffer für die Entwicklung von Burnout dienen könnten [16].

\section{Die aktuelle Studie}

In Anlehnung an die dargelegten theoretischen Überlegungen und empirischen Befunde wird in der vorliegenden Arbeit geprüft, inwieweit Annäherungszielorientierungen als personale Ressource in das SD-R-Modell eingeordnet werden können. Dazu wird zunächst getestet, ob sich die Annahmen des Modells in der vorliegenden Stichprobe replizieren lassen (H1, ESM 1). Anschließend wird der Zusammenhang zwischen studienbezogenen Ressourcen, Zielorientierungen und Engagement sowie dem Initialsymptom von Burnout, emotionaler Erschöpfung, untersucht (H2a, H2b, - Abb. 1). Hierbei werden Zielorientierungen als Mediator angenommen. Vor dem Hintergrund der skizzierten Befundlage werden demnach folgende Hypothesen formuliert:

- H1: Anforderungen und Ressourcen stehen in einer negativen Beziehung zueinander. Anforderungen korrelieren positiv mit Burnout und Engagement, während Ressourcen negativ mit Burnout und positiv mit Engagement korrelieren.

- H2a: Annäherungslern- und -leistungszielorientierungen mediieren

Präv Gesundheitsf 2022 · 17:1-6 https://doi.org/10.1007/s11553-021-00840-1

(c) Der/die Autor(en) 2021

T. Niewöhner · L. Hoerdler · A. Roxel · M. Meindl · Z. Teuber

\section{Zielorientierung, Burnout und Engagement im Hochschulkontext: Integration von Zielorientierung in das Study Demands- Resources-Modell}

Zusammenfassung

Hintergrund. Personale Ressourcen in Form motivationaler Faktoren haben in der bisherigen Forschung zum Thema Burnout im Hochschulkontext wenig Beachtung gefunden. Das Ziel der vorliegenden Studie war es, Annäherungslern- und -leistungsziele als personale Ressource in das etablierte Study Demands-Resources-Modell (SD-R-Modell) zu integrieren und ihren Zusammenhang mit Burnout sowie Engagement im Studium zu untersuchen.

Methodik. Mithilfe eines quantitativen Online-Fragebogens wurden Querschnittsdaten von 347 Studierenden $\left(M_{\text {Alter }}=22,9\right.$, $S D=3,2$ Jahre) verschiedener deutscher Hochschulen erhoben und mittels latenter Strukturgleichungsmodelle untersucht.
Ergebnisse. Die Ergebnisse zeigen, dass sich das SD-R-Modell replizieren lässt. Annäherungslernziele vermitteln den Zusammenhang zwischen Ressourcen und Engagement positiv. Entgegen der Hypothese vermitteln Annäherungslernziele den Zusammenhang zwischen Ressourcen und Burnout positiv. Leistungszielorientierung ist kein signifikanter Mediator.

Diskussion. Die Ergebnisse unterstreichen die Relevanz von Annäherungslernzielen für das Engagement und die psychische Gesundheit Studierender.

\section{Schlüsselwörter}

Motivation · Erschöpfung · Psychische Gesundheit · Studierende · Personale Ressourcen

\section{Goal orientation, burnout and engagement in higher education: integration of goal orientation into the Study Demands-Resources model}

\section{Abstract}

Background. Personal resources in the form of motivational factors have received little attention in the context of student burnout research. The present study integrates approach goal orientations as personal resources into the well-established Study Demands-Resources (SD-R) model and further investigates its relationship with burnout and study engagement.

Methods. The cross-sectional data of 347 students $\left(M_{\text {age }}=22.9, S D=3.2\right)$ from several German universities were collected by means of a quantitative online questionnaire and analyzed via structural equation modeling. Results. The results showed that the SD-R model could be replicated. Mastery- approach-orientation mediates the relation between resources and engagement positively. Against our assumptions, masteryapproach-orientation mediates the relation between resources and burnout positively. Performance-approach-orientation has no significant impact.

Conclusion. Results underline the importance of approach goal orientations for engagement and mental health of university students.

Keywords

Motivation - Exhaustion - Mental health . Students · Personal resources den Zusammenhang zwischen Ressourcen und Engagement positiv.

- H2b: Annäherungslern- und -leistungszielorientierung mediieren neben dem Zusammenhang zwischen Ressourcen und Engagement (positiv) auch den Zusammenhang zwischen Ressourcen und Burnout (negativ).
Für eine valide Überprüfung eines Mediationseffekts sind längsschnittliche $\mathrm{Da}$ ten notwendig. Die vorliegende Studie hat jedoch ein querschnittliches Design. Deshalb werden $\mathrm{H} 2 \mathrm{a}$ und $\mathrm{H} 2 \mathrm{~b}$ explorativ mit dem Ziel untersucht, Hinweise für spätere längsschnittliche Studien zu liefern. 
Tab. 1 Standardisierte Regressionsgewichte, Standardfehler und Signifikanzen

Beta SE P

\begin{tabular}{|c|c|c|c|}
\hline \multicolumn{4}{|l|}{ Modell 1} \\
\hline \multicolumn{4}{|l|}{ Burnout } \\
\hline Ressourcen & $-0,31$ & 0,09 & $<0,001$ \\
\hline Anforderungen & 0,46 & 0,10 & $<0,001$ \\
\hline \multicolumn{4}{|l|}{ Engagement } \\
\hline Ressourcen & 0,71 & 0,11 & $<0,001$ \\
\hline Anforderungen & $-0,06$ & 0,08 & 0,39 \\
\hline \multicolumn{4}{|l|}{ Modell 2} \\
\hline \multicolumn{4}{|l|}{ Burnout } \\
\hline Ressourcen & $-0,31$ & 0,09 & $<0,001$ \\
\hline Anforderungen & 0,46 & 0,10 & $<0,001$ \\
\hline \multicolumn{4}{|l|}{ Engagement } \\
\hline Ressourcen & 0,56 & 0,11 & $<0,001$ \\
\hline Anforderungen & $-0,04$ & 0,05 & 0,58 \\
\hline Annäherungslernziele & 0,36 & 0,09 & $<0,001$ \\
\hline Annäherungsleistungsziele & $-0,01$ & 0,04 & 0,77 \\
\hline \multicolumn{4}{|l|}{ Modell 3} \\
\hline \multicolumn{4}{|l|}{ Burnout } \\
\hline Ressourcen & $-0,37$ & 0,11 & $<0,001$ \\
\hline Anforderungen & 0,47 & 0,10 & $<0,001$ \\
\hline Annäherungslernziele & 0,15 & 0,09 & 0,05 \\
\hline Annäherungsleistungsziele & $-0,01$ & 0,06 & 0,87 \\
\hline \multicolumn{4}{|l|}{ Engagement } \\
\hline Ressourcen & 0,57 & 0,11 & $<0,001$ \\
\hline Anforderungen & $-0,04$ & 0,06 & 0,59 \\
\hline Annäherungslernziele & 0,35 & 0,09 & $<0,001$ \\
\hline Annäherungsleistungsziele & $-0,01$ & 0,04 & 0,77 \\
\hline
\end{tabular}

\section{Methodik}

\section{Stichprobe}

Im Frühjahr 2020 nahmen 503 Studierende an der Online-Fragebogenstudie teil. Nach der Bereinigung (z. B. viele fehlende bzw. ungültige Antworten) der Daten wurden Antworten von 347 Studierenden (davon 274 weiblich) in die Datenanalyse einbezogen. Das Durchschnittsalter betrug 22,9 $(S D=3,2)$ Jahre. Die Teilnehmenden waren Studierende im Bachelor $(n=217)$, Master $(n=120)$ sowie Promovierende $(n=4)$. Etwa $31 \%$ der Studierenden befanden sich zum Zeitpunkt der Erhebung im ersten Fachsemester und insgesamt $40 \%$ der Teilnehmenden gaben an, Psychologie zu studieren. Die Daten stammen insgesamt von Studierenden an 49 Universitäten und Fachhochschulen.
$64 \%$ der Teilnehmenden $(n=223)$ studierten an der Universität Bielefeld.

\section{Messinstrumente}

\section{Ressourcen}

Die Ressourcen wurden mit den Skalen zur sozialen sowie akademischen Integration und Studiumsfortschritten erfasst [4]. Es wurde die 5-stufige Likert-Subskala Emotionale Involviertheit mit 3 Items $(\alpha=0,84)$ verwendet.

\section{Zielorientierung}

Als personale Ressource wurde die Leistungszielorientierung mittels „Achievement Goal Questionnaire“ (AGQ-R; [7]) auf einer ebenfalls 5-stufigen Likert-Skala erhoben. Es wurden die Unterskalen $A n$ näherungslern- $(\alpha=0,81)$ und Annäherungsleistungsziele $(\alpha=0,91)$ mit jeweils 4 Items verwendet.
Studienbezogene Anforderungen

Für die Messung studienbezogener Anforderungen wurde das Berliner Anforderungs-Ressourcen-Inventar für Studierende (BARI-S; [9]) verwendet. Die eingesetzte Subskala in 6-stufiger Likert-Form erfasst die Überforderung im Studium mit 3 Items $(\alpha=0,64)$.

\section{Burnout}

Burnout wurde mittels der deutschen Kurzversion des Maslach-Burnout-Inventars für Studierende (MBI-SS KV; [24]) erhoben. Verwendet wurde die 6-stufige Subskala Emotionale Erschöpfung mit 3 Items $(\alpha=0,76)$.

\section{Studienbezogenes Engagement}

Studienbezogenes Engagement $(\alpha=0,79)$ wurde mit der 7-stufigen Utrecht Work Engagement Scale - Student Form Ultra Short (UWES-SF; [8]) erfasst. Das Inventar besteht aus 3 Items, die die drei Dimensionen Energie, Hingabe und Vitalität umfassen. Die Items sind in ESM 2 dargestellt.

\section{Analytische Strategien}

Mit dem Little's Test wurde überprüft, ob sich die Missings zufällig über die Datenstruktur erstreckten. Zur Prüfung der Hypothesen wurden drei latente Pfadmodelle aufgestellt, mittels des Pakets „lavaan“ [19] im Statistikprogramm R (Version 3.6.1) getestet und die Werte mit dem robusten Maximum-likelihoodVerfahren (Huber-White-Korrektur) geschätzt, welches robust gegenüber der Verletzung von Verteilungsannahmen ist. Modell 1 repliziert das SD-R-Modell (H1), Modell 2 führt die Mediation der studienbezogenen Ressourcen durch Zielorientierung auf das Engagement ein (H2a), Modell 3 erweitert Modell 2 um die Mediation zwischen Ressourcen und Burnout durch Zielorientierung (H2b). Bei allen Modellen wurde das Geschlecht als Kontrollvariable aufgenommen. Die Signifikanztestung unterlag einem Signifikanzniveau von $\alpha=0,05$. Bei den Modellvergleichen wurden die Richtwerte von $\mathrm{Hu}$ und Bentler [12] herangezogen. Auf Modelldifferenztestungen wurde entsprechend der Empfehlung von Urban und Mayerl [23] verzichtet. 


\begin{tabular}{|c|c|c|c|c|c|c|c|}
\hline Modell & $x^{2}$ & $D f$ & $p$ & RMSEA & CFI & AIC & BIC \\
\hline 1 & 135,545 & 48 & $<0,001$ & 0,072 & 0,937 & $12.259,611$ & $12.421,282$ \\
\hline 2 & 252,510 & 125 & $<0,001$ & 0,054 & 0,951 & $17.307,801$ & $17.554,157$ \\
\hline 3 & 248,871 & 123 & $<0,001$ & 0,054 & 0,952 & $17.307,077$ & $17.561,132$ \\
\hline
\end{tabular}

\section{Ergebnisse}

Das ESM 3 präsentiert Mittelwerte, Standardabweichungen und Interkorrelationen der Skalen. - Tab. 1 zeigt die standardisierten Regressionsgewichte, Standardfehler und Signifikanzen der latenten Regressionspfade.

Wie erwartet (H1), korrelierten Anforderungen und Burnout sowie studienbezogene Ressourcen und Engagement positiv. Hypothesenkonform korrelierten studienbezogene Ressourcen und Burnout negativ. Lediglich der Zusammenhang zwischen Anforderungen und Engagement konnte nicht nachgewiesen werden. Insgesamt wurden $38 \%$ der Varianz in Burnout und $52 \%$ der Varianz im Engagement erklärt.

Hypothesenkonform ( $\mathrm{H} 2 \mathrm{a})$ mediierte die Lernzielorientierung den Zusammenhang zwischen studienbezogenen Ressourcen und Engagement positiv. Höhere Lernziele hingen mit höherem Engagement zusammen. Entgegen der Hypothese ist die Leistungszielorientierung kein signifikanter Mediator. Es konnten $38 \%$ der Varianz in Burnout und $62 \%$ der Varianz in Engagement erklärt werden.

Entgegen der Hypothese (H2b) war die Leistungszielorientierung kein signifikanter Mediator zwischen studienbezogenen Ressourcen und Burnout. Lernzielorientierung mediierte den Zusammenhang zwischen Ressourcen und Engagement, jedoch entgegen der erwarteten Wirkrichtung. Höhere Lernziele hingen mit höherem Burnout zusammen. Modell 3 erklärte $41 \%$ der Varianz in Burnout und $62 \%$ der Varianz in Engagement.

In • Tab. 2 ist die Modellgüte der drei Modelle angegeben. Aufgrund der Gütekriterien und dem Anteil der aufgeklärten Varianz kann Modell 2 angenommen werden.

\section{Diskussion}

Ziel dieser Studie war es, die Anwendbarkeit des im Hochschulkontext etablierten SD-R-Modells um die Einflussnahme der personalen Ressourcen in Form von Zielorientierungen zu erweitern. Das Modell wurde damit erstmalig mit der erweiterten Komponente der Zielorientierung in Deutschland untersucht.

Sowohl der motivationale Pfad zwischen Ressourcen und Engagement als auch der gesundheitsbeeinträchtigende Pfad zwischen Anforderungen und Burnout konnten durch die vorliegende Studie repliziert werden.

Im Kontrast zu bisherigen Anwendungen des SD-R-Modells wurde das Wirkgefüge des Modells um die Annäherungslern- und -leistungsziele als Mediator zwischen den studienbezogenen Ressourcen, dem Engagement sowie Burnout erweitert (Modell 2 und 3). Die Ergebnisse unterstreichen die $\mathrm{Me}$ diatorrolle von Annäherungslern- und -leistungszielen.

Im Arbeitskontext konnten darüber hinaus bereits Jobressourcen wie Kontroll- und Entscheidungsfreiheiten bei der Arbeitsausführung oder die Möglichkeit zur beruflichen Weiterentwicklung als Mediator zwischen personalen Ressourcen und Arbeitsengagement identifiziert werden [25]. Hier dienten also die Jobressourcen als Mediator. Dies spricht insgesamt für einen reziproken Zusammenhang von Arbeitscharakteristika und personalen Faktoren, der von Xanthopoulou et al. [26] bereits bestätigt werden konnte. Die Möglichkeit wechselseitiger Zusammenhänge nehmen Bakker et al. [2] in ihrer JD-R-Theorie auf. Deshalb sollte diese Möglichkeit im Hochschulkontext in weiteren Studien geprüft werden.

Darüber hinaus stellt die vorliegende Studie die Relevanz von Annäherungs- zielorientierungen heraus, indem sie an die Ergebnisse von Naidoo et al. [16] anknüpft. Es konnten sowohl zwischen Annäherungslern- und -leistungszielen positive Zusammenhänge mit dem Engagement der Studierenden und negative $\mathrm{Zu}$ sammenhänge mit Burnout festgestellt werden. Ein wichtiger Befund ist hierbei, dass eine mittlere Fähigkeitsausprägung bei Annäherungslernzielen das Engagement der Studierenden optimal beeinflusst. Ist die Zielsetzung jedoch hoch ausgeprägt, so dass ausschließlich überhöhte Ziele gesetzt werden, kann dies einen Kontrasteffekt hervorrufen und sich negativ auf das Engagement auswirken und das Burnout-Risiko erhöhen. Dieser Befund zeigt einen neuen Ansatzpunkt, der in bisherigen Untersuchungen noch unentdeckt blieb und in den Fokus weiterer Forschung gesetzt werden sollte.

Die wesentliche Limitation dieser Studie ist das Querschnittsdesign. Es ist zusätzlich erforderlich längsschnittliche Daten $\mathrm{zu}$ erheben, um kausale Interpretationen der Effekte (insbesondere der Mediationseffekte) vornehmen $\mathrm{zu}$ können. Dennoch wurde das JD-R-Modell auch schon in längsschnittlichen Designs untersucht, was die Annahmen unterstützt [26]. Weiterhin sollte bedacht werden, dass die Studie nur auf selbstbeschreibenden Messdaten beruht und keine objektiven Daten erhoben wurden. Methodische Bias sind somit nicht auszuschließen [13]. Außerdem sollte die Zusammensetzung der Stichprobe beachtet werden. Die Daten stammen größtenteils von einer Hochschule und Studiengruppe. Die Ergebnisse sind daher für deutsche Studierende nicht repräsentativ. Außerdem werden Studierende aus Bachelor- und Masterstudiengängen gemeinsam betrachtet. Damit folgt diese Arbeit vorangegangenen Studien zum SD-R-Modell (z. B. [10]). Zukünftige Arbeiten sollten eine Analyse, getrennt nach Fortschritt im Studium (z.B. Bachelor vs. Master) anstreben, um möglicherweise fortschrittsbedingte Einflüsse von Zielorientierungen auf Engagement und Burnout aufzudecken.

Zusammenfassend lässt sich festhalten, dass das bisher angenommene SD-RModell repliziert und durch die Annäherungslern- und -leistungsziele als perso- 
nale Ressource erweitert werden konnte. Insgesamt zeigen die Ergebnisse, dass besonders die Annäherungslernziele eine wichtige Komponente im SD-R-Modell darstellen, da sie den Zusammenhang zwischen studienbezogenen Ressourcen und Engagement sowie Burnout vermitteln.

\section{Fazit für die Praxis}

- Die Befunde deuten darauf hin, dass die Zielorientierungen der Studierenden ein wichtiger Ansatzpunkt für das Gesundheitsmanagement von Hochschulen (insbesondere der Studienberatung) sein sollte, um das Engagement im Studium zielgerichtet zu steigern und Burnout zu vermeiden.

- Die Befunde implizieren zudem, dass zu hohe Annäherungslernziele zu überhöhten Zielen führen können, was wiederrum einen positiven Zusammenhang mit Burnout erklärt.

- Das Ergebnis unterstreicht, dass die Differenzierung zwischen der Dimension Lern- und Leistungsziel in der Praxis von Bedeutung ist.

\section{Korrespondenzadresse}

Thomas Niewöhner
Fakultät für Psychologie
und Sportwissenschaften,
Abteilung für Psychologie,
Universität Bielefeld
Universitätsstraße 25,
33615 Bielefeld, Deutschland
thomas.niewoehner@
uni-bielefeld.de

Funding. Open Access funding enabled and organized by Projekt DEAL.

\section{Einhaltung ethischer Richtlinien}

Interessenkonflikt. T. Niewöhner, L. Hoerdler, A. Roxel, M. Meindl und Z. Teuber geben an, dass kein Interessenkonflikt besteht.

Alle beschriebenen Untersuchungen am Menschen wurden mit Zustimmung der zuständigen Ethik-Kommission der Universität Bielefeld durchgeführt. Von allen beteiligten Studierenden liegt eine Einverständniserklärung vor. Es besteht kein Interessenkonflikt der Autoren.
Open Access. Dieser Artikel wird unter der Creative Commons Namensnennung 4.0 International Lizenz veröffentlicht, welche die Nutzung, Vervielfältigung, Bearbeitung, Verbreitung und Wiedergabe in jeglichem Medium und Format erlaubt, sofern Sie den/die ursprünglichen Autor(en) und die Quelle ordnungsgemäß nennen, einen Link zur Creative Commons Lizenz beifügen und angeben, ob Änderungen vorgenommen wurden.

Die in diesem Artikel enthaltenen Bilder und sonstiges Drittmaterial unterliegen ebenfalls der genannten Creative Commons Lizenz, sofern sich aus der Abbildungslegende nichts anderes ergibt. Sofern das betreffende Material nicht unter der genannten Creative Commons Lizenz steht und die betreffende Handlung nicht nach gesetzlichen Vorschriften erlaubt ist, ist für die oben aufgeführten Weiterverwendungen des Materials die Einwilligung des jeweiligen Rechteinhabers einzuholen.

Weitere Details zur Lizenz entnehmen Sie bitte der Lizenzinformation auf http://creativecommons.org/ licenses/by/4.0/deed.de.

\section{Literatur}

1. Bakker AB, Demerouti E (2014) Job demandsresources theory. In: Cooper $\mathrm{CL}$, Chen PY (Hrsg) Wellbeing. Wiley \&Sons, Chichester, S1-28 https:// doi.org/10.1002/9781118539415

2. Bakker AB, Demerouti E, Sanz-Vergel AI (2014) Burnout and work engagement: the JD-R approach. Annu Rev Organ Psychol Organ Behav 1(1):389-411. https://doi.org/10.1146/annurevorgpsych-031413-091235

3. Bound J, Turner S (2011) Dropouts and diplomas: the divergence in collegiate outcomes. Handbook of the economics of education, Bd. 4, S 573-613 https://doi.org/10.1016/B978-0-444-53444-6. 00008-0

4. Dahm G, Lauterbach O, Hahn S (2016) Measuring students' social and academic integrationassessment of the operationalization in the national educational panel study. In: Blossfeld H-P von Maurice J, Bayer M, Skopek J (Hrsg) Methodol. Issues Longitud. Surv. Springer, Wiesbaden, S 313-329

5. Elliot AJ (1999) Approach and avoidance motivation and achievement goals. Educ Psychol 34(3):169-189. https://doi.org/10.1207/ s15326985ep3403_3

6. Elliot AJ, McGregor HA (2001) A 2×2 achievement goal framework. J Pers Soc Psychol 80(3):501-519. https://doi.org/10.1037//0022-3514.80.3.501

7. Elliot AJ, Murayama K (2008) On the measurement of achievement goals: critique, illustration, and application. J Educ Psychol 100(3):613-628. https://doi.org/10.1037/0022-0663.100.3.613

8. Gusy B, Lesener T, Wolter C (2019) Measuring well-being with the Utrecht work engagement scale-student form. Eur J Health Psychol 26(2):31-38. https://doi.org/10.1027/2512-8442/ a000027

9. Gusy B, Lohman K (2011) Gesundheit im Studium Dokumentation der Instrumente (Schriftenreihe des AB Public Health: Prävention und psychosoziale Gesundheitsforschung Nr. 01/P11). Freie Universität Berlin, Berlin

10. Gusy B, Wörfel F, Lohmann K (2016) Erschöpfung und Engagement im Studium. Z Gesundhpsycho 24:41-53. https://doi.org/10.1026/0943-8149/ a000153
11. Hobfoll SE, Johnson RJ, Ennis N, Jackson AP (2003) Resource loss, resource gain, and emotional outcomes among inner city women. J Pers Soc Psychol 84(3):632-643. https://doi.org/10.1037/ 0022-3514.84.3.632

12. Hu L, BentlerPM (1999)Cutoff criteria for fit indexes in covariance structure analysis: conventional criteria versus new alternatives. Struct Equ Modeling 6(1):1-55. https://doi.org/10.1080/ 10705519909540118

13. Lesener T, Pleiss LS, Gusy B, Wolter C (2020) The study demands-resources framework: an empirical introduction. Int J Environ Res Public Health 17(14):5183. https://doi.org/10.3390/ ijerph17145183

14. Maske UE, Riedel-Heller SG, Seiffert I et al (2016) Häufigkeit und psychiatrische Komorbiditäten von selbstberichtetem diagnostiziertem BurnoutSyndrom. Psychiatr Prax 43:18-24. https://doi.org/ 10.1055/s-0034-1387201

15. MiddendorffE, Apolinarski B, Becker Ket al (2017) Die wirtschaftliche und soziale Lage der Studierenden in Deutschland 2016. Zusammenfassung zur 21. Sozialerhebung des Deutschen Studentenwerks - durchgeführt vom Deutschen Zentrum für Hochschul-und Wissenschaftsforschung

16. Naidoo LJ, DeCriscio A, Bily $H$, Manipella A et al (2012) The $2 \times 2$ model of goal orientation and burnout: the role of approach-avoidance dimensions in predicting burnout. J Appl Soc Psychol 42(10):2541-2563. https://doi.org/10. 1111/j.1559-1816.2012.00952.x

17. Pfleging S, Gerhardt C (2013) Ausgebrannte Studierende: Burnout-Gefährdung nach dem Bologna-Prozess. JBus Psychol 4(1):1-12

18. Reschly AL, Christenson SL (2012) Jingle, jangle, and conceptual haziness: evolution and future directions of the engagement construct. In: Christenson SL, Reschly AL, Wylie C (Hrsg) Handbook of research on student engagement. Springer US, Boston, MA, S3-19

19. Rosseel Y (2011) lavaan: an R package for structural equation modeling. J Stat Softw. https://doi.org/ 10.18637/jss.v048.i02

20. Schaufeli WB, Salanova M, González-Romá V, Bakker $A B$ (2002) The measurement of engagement and burnout: a two sample confirmatory factor analytic approach. J Happiness Stud 3(1):71-92. https://doi.org/10.1023/A:1015630930326

21. Shih S-S (2012) An examination of academic burnout versus work engagement among Taiwanese adolescents. J Educ Res 105(4):286-298. https:// doi.org/10.1080/00220671.2011.629695

22. Tuominen-Soini $H$, Salmela-Aro K, Niemivirta M (2008) Achievement goal orientations and subjective well-being: a person-centred analysis. Learn Instr 18(3):251-266. https://doi.org/10. 1016/j.learninstruc.2007.05.003

23. Urban D, Mayerl J (2014) Strukturgleichungsmodellierung. Springer, Wiesbaden

24. Wörfel F, Gusy B, Lohman K, Kleiber D (2015) Validierung der deutschen Kurzversion des Maslach-Burnout-Inventars für Studierende (MBISS KV). Z Gesundhpsychol 23(4):191-196. https:// doi.org/10.1016/j.learninstruc.2007.05.003

25. Xanthopoulou D, Bakker AB, Demerouti E, Schaufeli WB (2007) The role of personal resources in the job demands-resources model. Int J Stress Manag 14(2):121-141. https://doi.org/10.1037/ 1072-5245.14.2.121

26. Xanthopoulou D, Bakker AB, Demerouti E, Schaufeli WB (2009) Reciprocal relationships between job resources, personal resources, and work engagement. J Vocat Behav 74(3):235-244. https://doi.org/10.1016/j.jvb.2008.11.003 